\title{
ESTIMATIVA DO SEXO E IDADE POR MEIO DO ÍNDICE TRANSVERSO EM CRÂNIOS SECOS DE ADULTOS
}

\author{
Erasmo de Almeida Júnior \\ Professor Associado da disciplina Anatomia Humana \\ da Universidade Federal da Bahia. \\ Francisco Prado Reis \\ Luis Carlos Cavalcante Galvão \\ Professor titular da disciplina de Neuroanatomia da \\ Universidade Tiradentes. \\ Professor adjunto da disciplina de Odontologia Legal \\ da Universidade Federal da Bahia. \\ Marcelo Correa Alves \\ Doutor em Anatomia Humana pela Universidade de \\ São Paulo. \\ Adriana Rúbia de Jesus Cabral \\ Cirurgiã Dentista pela Universidade Federal de \\ Alagoas. \\ Sheila Teixeira

\begin{abstract}
Acadêmica do curso de Odontologia da União
\end{abstract} \\ Metropolitana para o Desenvolvimento da Educação \\ e Cultura.
}

Endereço para correspondência: anatjjunior@ig.com.br

\begin{abstract}
Resumo
Introdução - A Odontologia Legal tem grande importância no processo de identificação, principalmente quando os profissionais da área recebem para análise apenas a porção cefálica do corpo. O objetivo deste estudo é verificar o dimorfismo sexual e estimação da idade através do Índice Transverso Craniano. Material e Métodos - Os autores estudaram uma amostra de 160 crânios secos, sendo 80 masculinos e 80 femininos, que pertenceram a indivíduos com idade acima de 20 anos, e com sexo e idade conhecidos com absoluta segurança. Os crânios estudados eram de pessoas cujos familiares não reclamaram os ossos no tempo hábil administrativo estabelecido pelo Cemitério e que estavam sendo encaminhados para incineração. Foram realizadas as seguintes mensurações: distância básio-bregma (altura craniana máxima) e distância éurio-éurio (largura craniana máxima) e a partir destas foi calculado o Índice Transverso. Resultados - De acordo com a análise estatística, houve índice de acerto de $81,5 \%$ por regressão logística e $76,25 \%$ pela análise discriminante. Foi também verificada a média e intervalo de confiança através do teste t e utilizado o método da regressão linear múltipla para a predição da idade, constatando que o modelo não foi significativo $(\mathrm{p}=0,3223)$. Conclusões - Os resultados permitiram a elaboração de metodologia estatística para o diagnóstico do sexo e idade em observações futuras.
\end{abstract}

Palavras chave: Odontologia legal; Sexo; Idade; Crânio.

\section{ESTIMATION OF SEX AND AGE THROUGH THE TRANSVERSE INDEX IN DRY ADULT SKULLS}

\begin{abstract}
Introduction - Forensic Dentistry has great importance in the identification process, especially when professionals receive only the cephalic portion of the body for analysis. The purpose of this study is to assess sexual dimorphism and age estimation using the Transverse Cranial Index. Materials and methods - We studied a sample of 160 dry skulls, 80 males and 80 females, who belonged to individuals over the age of 20 years, and with age and sex known with absolute certainty. The studied skulls belonged to people whose relatives had not claimed their remains in the time established by the Cemetery administration and they were
\end{abstract}


being sent for cremation. The following measurements were carried out: the distance between the basion and bregma (maximum cranial height) and the distance between the eurio and eurio (maximum cranial width) and from these we calculated the Transverse Index. Results - According to statistical analysis, there was a success rate of $81.5 \%$ by logistic regression and $76.25 \%$ with discriminant analysis. The results were verified and the confidence interval was average throughout the test. The method of multiple linear regression used to predict the age was used, noting that the model was not significant $(\mathrm{p}=0.3223)$. Conclusions - The results allowed the development of statistical methodology for the diagnosis of sex and age on future observations.

Keywords: Forensic dentistry; Sex; Age; Skull.

\section{INTRODUÇÃO}

O processo de identificação de pessoas, a partir de exames periciais, do segmento cefálico ou de partes dele, tem sido de significante importância para o esclarecimento de fatos de interesse jurídico-social. A tarefa exige técnicas e métodos mais precisos que venham auxiliar peritos médicos e odontólogos legistas a desempenharem eficientemente seu trabalho de auxiliar cientificamente a justiça quer no direito civil, penal, do trabalho e, em certos casos, até no direito administrativo. ${ }^{(1)}$

O capítulo da identificação, considerado uma das mais importantes funções do perito Médico e Odontolegal, é muito vasto e complexo, não podendo ser confundido com reconhecimento. Este último trata-se de um procedimento empírico baseado apenas em conhecimento prévio, cuja base de sustentação é unicamente testemunhal. ${ }^{(2)}$

A investigação do crânio, em algumas situações, pode fornecer elementos importantes para a identificação do sexo de uma pessoa. Essas situações podem se tratar de um indivíduo vivo, cadáver cronologicamente recente, cadáver em processo de putrefação ou de esqueletização, carbonizados, esqueleto completo ou partes dele, como por exemplo o crânio. ${ }^{(3,4)}$

A estimativa do sexo é um dos quatro pilares do protocolo antropológico, que consiste da análise métrica e avaliação visual das características do esqueleto, do crânio e da pelve. Desse modo, quanto mais mensurações e dados forem observados num laudo, mais confiável será o resultado. ${ }^{(2,5)}$

Qualitativamente, em geral, os crânios masculinos apresentam as estruturas mais grosseiras ou ásperas, devido às inserções musculares serem mais fortes. Dentre essas estruturas, podem ser citadas: a glabela, processos mastóides, rebordo orbitário, palato, 
abertura piriforme, extensão zigomática e rugosidades supraorbital. ${ }^{(6)}$

Quantitativamente, os crânios masculinos são também, em geral, maiores do que os femininos, podendo-se relatar algumas características como: capacidade craniana, peso, diâmetro ântero-posterior, diâmetro transverso, altura craniana, largura bizigomática e espessura óssea. ${ }^{(7,8)} \mathrm{O}$ sexo do indivíduo pode ser facilmente estimado com o uso de um pequeno conjunto de 11 variáveis craniométricas. ${ }^{(9)}$

O exame do dimorfismo sexual através do crânio tem sido objeto de numerosos estudos morfológicos e craniométricos, mas a desvantagem desses estudos é que eles são dependentes da experiência do operador. $\mathrm{Na}$ verdade, o grande problema que envolve a Antropologia Forense é a variabilidade morfológica e métrica que ocorre em populações diferentes, devido a fatores climáticos, alimentares e sócio-organizacionais, sendo necessária a realização de mais estudos em nossa população. ${ }^{(10)}$

O presente estudo é uma tentativa de verificar o dimorfismo sexual e estimar a idade, examinando crânios secos através da medida das distâncias entre os seguintes pontos craniométricos: básio-bregma (abb), éurio-éurio (dtm) e Índice Transverso (it).

\section{MATERIAL E MÉTODO}

Foram utilizados para o estudo 160 crânios secos, 80 do sexo feminino e 80 masculino, todos maiores de 20 anos de idade. Os crânios foram obtidos de acordo com a lei $\mathrm{n}^{\circ} 8501$ de 1992, que trata do uso de cadáveres não reclamados para uso em estudos e pesquisas. Os crânios tinham sexo e idade registrados no Cemitério Público Quinta dos Lázaros, localizado na cidade de Salvador, estado da Bahia, Brasil, local onde também foram realizadas as mensurações dos crânios. Foram incluídos na pesquisa os crânios que não apresentavam qualquer tipo de dano, ou variações anatômicas relacionados à região de estudo. Não foram levados em consideração dados socioeconômico e cor da pele.

Para a obtenção das medidas foi utilizado um compasso de espessura da marca Vonder, uma régua milimetrada devidamente calibrada e uma tábua osteométrica confeccionada em acrílico também graduada em milímetro e devidamente calibrada. Foram tomadas medidas lineares que correspondiam às distâncias entre os seguintes pontos craniométricos: básio-bregma (básio é o ponto localizado na borda anterior do forame 
magno e bregma é o ponto de encontro entre as suturas coronária e sagital); éurio-éurio (éurio é o ponto localizado no extremo do diâmetro transverso máximo, sobre a protuberância parietal). A partir destas medidas foi calculado o Índice Transverso através da seguinte fórmula:

$$
\text { it }=\frac{\text { Altura mảxima }(a b b)}{\text { Largura mäxima }(\text { dtm })} \times 100
$$

A análise estatística foi realizada utilizando-se os seguintes métodos: para predição do sexo foi utilizado o teste t, para comparação das médias e intervalo de confiança além da regressão logística, análise discriminante e o método da regressão linear múltipla, para estimativa da idade. Em todos os testes utilizados foi adotado um nível de significância de $5 \%$ e os cálculos foram realizados com o uso do sistema SAS.

\section{RESULTADOS}

Os resultados obtidos na pesquisa de campo foram agrupados e submetidos à análise estatística, apresentando os seguintes resultados:

\subsection{Caracterização da amostra}

A amostra foi caracterizada com base na comparação das médias das variáveis estudadas de cada sexo, cujos valor médio, desvio padrão e limites de intervalos de confiança estão representados na Tabela 1. A análise de variância dos dados mostrou que os indivíduos do sexo masculino apresentaram médias das variáveis maiores que as do sexo feminino. De acordo com o teste $\mathrm{t}$, ocorreu diferença significativa entre as médias das variáveis exceto na variável it, que ficou um pouco acima do limite de confiança. Os intervalos de confiança das variáveis $a b b$ e $d t m$ foram desconexos, ou seja, não houve interposição de faixas, indicando que cada uma das variáveis apresentou bons indícios para discriminação do sexo a partir das medidas realizadas. Com relação a variável $i t$, houve uma pequena interposição de faixa, o que não prejudicou em nada a aplicação de outros 
métodos estatísticos mais específicos.

Tabela 1 - Estatísticas básicas (média, desvio padrão e limites dos intervalos de confiança da média - 95\%) das variáveis observadas (n: 160) e teste t para comparação das médias das variáveis abb, dtm e it nos diferentes sexos.

Limite do intervalo de

confiança da média $(95 \%)$

\begin{tabular}{llllrl} 
Variável & & & Desvio & \\
\cline { 5 - 6 } & Sexo & Média $(\mathrm{mm})$ & padrão & superior & inferior \\
\hline$a b b$ & Feminino & 126,513 & 5,558 & 127,749 & 125,276 \\
valor-p: $<0,0001$ & Masculino & 132,638 & 7,568 & 134,322 & 130,953 \\
\hline$d t m$ & Feminino & 137,725 & 6,100 & 139,083 & 136,367 \\
valor-p: 0,0002 & Masculino & 141,400 & 6,033 & 142,743 & 140,057 \\
\hline$i t$ & Feminino & 91,965 & 5,189 & 93,120 & 90,810 \\
valor-p: 0,0563 & Masculino & 93,949 & 7,629 & 95,647 & 92,251 \\
\hline
\end{tabular}

Fonte: Elaboração dos autores.

\subsection{Análise Discriminante}

Para a análise discriminante, foram construídas duas equações a partir dos dados encontrados, sendo que o resultado maior entre as duas equações corresponde ao sexo indicado:

$$
\begin{aligned}
\text { Feminino } & =-25821-388,82431 \times a b b+366,17645 \times d t m+548,04833 \times \text { it } \\
\text { Masculino } & =-25873-388,80208 \times a b b+366,41003 \times d t m+548,22620 \times \text { it }
\end{aligned}
$$

A avaliação da capacidade de discriminação foi obtida através de um processo de ressubstituição ou reclassificação no qual os dados conduziram a uma estimativa do sexo. Pelo processo de ressubstituição, foram encontrados índices de acertos da ordem de 75,0\% em dados do sexo feminino e de $77,5 \%$ para os do sexo masculino, apresentando uma taxa total de erros da ordem de $23,75 \%$, inferior àquela obtida em dependência do acaso, que seria de $50 \%$ (Tabela 2). 
Tabela 2 - Ressubstituição contrapondo os sexos observados e preditos através das funções lineares discriminantes a partir dos dados que foram usados na estimativa dos parâmetros do modelo (n: 160).

\begin{tabular}{lrrr}
\hline \multirow{2}{*}{ Sexo observado } & \multicolumn{2}{c}{ Sexo predito } & Total \\
\cline { 2 - 4 } Feminino & Feminino & Masculino & 80 \\
& 60 & 20 & 100,00 \\
Masculino & 75,00 & 25,00 & 80 \\
& 18 & 62 & 100,00 \\
\hline \multirow{2}{*}{ Total } & 22,50 & 77,50 & 160 \\
& 78 & 82 & 100,00 \\
Porcentagem de erros & 48,75 & 51,25 & 23,75 \\
Acertos ao acaso & 25,00 & 22,50 & \\
\hline
\end{tabular}

Fonte: Elaboração dos autores.

\subsection{Regressão logística}

$\mathrm{Na}$ regressão logística, partindo do estudo de seleção das variáveis através do método Stepwise, foi verificado que para apoiar a decisão de seleção do sexo seria adequado o uso de duas dentre as três variáveis medidas: as distâncias básio-bregma (abb) e distância éurio-éurio (dtm).

Através dos dados encontrados, foi elaborada a seguinte equação:

$$
\text { Logito }=39,5353-0,1791 \times a b b-0,1172 \times d t m
$$

Este modelo foi considerado altamente significativo e permitiu estimar a pertinência de uma medida ao sexo feminino. A transformação deste logito na probabilidade de pertinência da medida à pessoas do sexo feminino foi obtida através da seguinte função:

$p f=e^{39,5353-0,1791 \times a b b-0,1172 \times d t m} /(1+e)^{39,5353-0,1791 \times a b b-0,1172 \times d t m}$

onde: $e=2,71828$

Através desta equação, foi atingido um índice de concordância de 81,5\%. Os 
parâmetros de correlação indicaram valores em torno de 63,0\% (Tabela 3).

Tabela 3. Associação entre probabilidades estimadas e respostas observadas.
Percentagem de concordância $\quad 81,5$
D de Somer : $\quad 63,3$

Percentagem de discordância $\quad 18,2$

: $\quad$ Gamma: 63,5

Percentagem de empate : 0,3

Tau-a: $\quad 0,319$

Pares : 6400

c : 0,817

Fonte: Elaboração dos autores.

\section{4 Regressão Linear Múltipla}

A predição da idade foi realizada através de um modelo de regressão linear múltipla, que foi iniciado através da análise de variância do modelo de regressão. De acordo com os dados, foi constatado que o modelo de predição da idade não foi significativo (p: 0,3223), demonstrado na Tabela 4.

Tabela 4 - Análise de variância do modelo de regressão linear múltipla para predição das idades em função das variáveis

\begin{tabular}{lrrrcl}
\hline & & Soma de & Quadrados & \\
Causa de variação & GL & quadrados & médios & Valor F & Valor-p \\
Modelo & 1 & 252,50625 & 252,50625 & 0,99 & 0,3223 \\
Resíduo & 158 & 40465 & 256,10562 & & \\
Total corrigido & 159 & 40717 & & & \\
\hline
\end{tabular}

Fonte: Elaboração dos autores.

\section{DISCUSSÃO}

Excetuando-se a pelve, o crânio é amplamente considerado o melhor indicador para o diagnóstico do sexo. ${ }^{(11)} \mathrm{Na}$ grande maioria dos estudos a respeito da identificação do sexo de um indivíduo, a partir do exame da porção cefálica, tem sido utilizado o método qualitativo. São frequentemente estudadas características tais como: os seios frontais, dentes, glabela, espessura dos ossos dos arcos superciliares, forma do mento, aspecto da superfície óssea devido à ação da musculatura, processos mastóides, eminências parietais, 
fossa canina, arcos alveolares, processos coronóides e articulação frontonasal. ${ }^{(1,6)}$

Em estudos são utilizadas medidas entre pontos pré-estabelecidos, que proporcionam critérios mais seguros na questão do diagnóstico do sexo. Muitos autores têm contribuído de maneira clara e científica para a determinação do sexo através de métodos quantitativos. $^{(3,8,12,13,14)}$ Essa literatura, na sua maioria, utilizou amostras estrangeiras, fato que tem limitado sua aplicabilidade à população brasileira.

Com relação a mandíbula, é elevado o grau de dimorfismo sexual, especialmente a nível de seu ramo mandibular que, normalmente, devido ao processo de mastigação é submetido a maior estresse do que qualquer outro osso do crânio. ${ }^{(15)}$ No presente estudo, as medidas das distâncias básio-bregma (abb), éurio-éurio (dtm) e Índice Transverso (it), comparadas ao que se depreende entre os métodos qualitativos, pareceram mais eficientes, visto terem fornecido valores matemáticos que, analisados estatisticamente, podem contribuir para a redução ou a eliminação de erros de observação, interpretação pessoal e deformação anatômica relativa. O Teste t, utilizado no presente estudo, por exemplo, forneceu resultados satisfatórios. Outros autores também obtiveram bons resultados com relação a este método. ${ }^{(2,12,13,14)}$

A regressão logística tem sido empregada em estudos semelhantes por outros autores. ${ }^{(2,16)}$ Através de uma seleção de variáveis identificadas pelo método Stepwise, foi constatado que seria adequado o uso de duas dentre as três variáveis medidas para apoiar a decisão de seleção do sexo: $a b b$ e $d t m$. A partir deste método, obtemos um índice de concordância de 81,5\%. Alguns autores, utilizando o mesmo tratamento estatístico, chegaram também a resultados considerados satisfatórios: Galvão(1994) ${ }^{(12)}$ obteve 93,8\% de acerto e em estudo posterior (1998) ${ }^{(2)}$ encontrou índices de 92,9\% e 94,7\% para os sexos feminino e masculino, respectivamente. Saliba $(1999)^{(13)}$ e Sampaio (1999) ${ }^{(14)}$ também, utilizando a regressão logística, obtiveram resultados satisfatórios.

Quanto à análise discriminante empregada também por alguns autores, ${ }^{(2,12,13,14)}$ no presente estudo foi encontrado um índice de acerto de 75,0\% em dados do sexo feminino e $77,5 \%$ para dados masculinos, com uma taxa total de erro de $23,75 \%$, sendo inferior àquela obtida ao acaso, que seria de 50\%. Com relação a predição da idade, em que foi utilizado o método da regressão linear múltipla, o modelo não foi considerado significativo, 
apresentando um valor de $\mathrm{p}=0,3223$.

Os dados obtidos no presente estudo permitiram concluir que a medida das variáveis estudadas foram menor em crânios do sexo feminino. Este resultado está de acordo com alguns autores, que em seus estudos avaliaram áreas faciais quantitativamente. ${ }^{(14,17,18)} \mathrm{A}$ utilização de diferentes métodos estatísticos teve o objetivo de diminuir a margem de erro. A utilização desses métodos ofereceu maior confiabilidade nos resultados, quando da aplicação nos casos de identificação, podendo ser utilizados em serviços de Antropologia Forense e nos Institutos Médicos Legais. Considerando que o presente estudo utilizou uma amostra nacional, pode ser admitido que isto venha evitar alguns dos inconvenientes apresentados pelas amostras estrangeiras resultante de fatores tais como: clima, alimentação, condição socioeconômica e qualidade de vida, que poderão possivelmente interferir no diagnóstico.

\section{CONCLUSÕES}

A metodologia realizada para a investigação do sexo através de medida de distâncias entre pontos da face, de acordo com os resultados da técnica da regressão logística, com a amostra utilizada, teve um acerto de 81,5\%. A amostra foi também, durante a investigação, submetida à análise de função discriminante, por média e intervalo de confiança. Quanto à investigação da idade, o modelo do estudo não apresentou significância estatística, não demonstrando, portanto, confiabilidade. É possível que metodologias como a empregada no presente estudo, isolada ou ao lado de outras, possam vir a contribuir para o acerto de pesquisas sobre a investigação do sexo dentro da medicina legal e de outros campos de estudo afins.

\section{Referências}

1. França GV. Medicina Legal. $5^{a}$ ed. Rio de Janeiro: Guanabara Koogan; 1998.

2. Galvão LCC. Determinação do sexo através da curva frontal e apófise mastoide. [tese] São Paulo: Faculdade de Odontologia de Piracicaba da Universidade Estadual de Campinas; 1998. 
3. Francesquini Junior L, Francesquini LA, De La Cruz BM, Prereira SD, Ambrosano GM, Barbosa CM et al. Identification of sex using cranial base measurements. J Forensic Odontostomatol. 2007;25(1):7-11.

4. Patil KR, Mody RN. Determination of sex by discriminant function analysis and stature by regression analysis: a lateral cephalometric study. Forensic Sci Int. 2005;147(2-3): 175-80.

5. Kimmerle EH, Ross A, Slice D. Sexual dimorphism in America: geometric morphometric analysis of the craniofacial region. J Forensic Sci. 2008;53(1):54-7.

6. Rogers T L. Determing the sex of human remains through cranial morphology. J Forensic Sci. 2005;50(3):493-500.

7. Gapert R, Black S, Last J. Sex determination from the foramen magnum: discriminant function analysis in an eighteenth and nineteenth century British sample. Int $\mathbf{J}$ Legal Med. 2009;123(1):25-33.

8. Hatipoglu HG, Ozcan HN, Hatipoglu US, Yuksel E. Age, sex and mass index in relation to calvarial diploe thickness and craniometric data on MRI. Forensic Sci Int. 2008; 182(1-3):46-51.

9. Konigsberg IW, Algee-Hewitt BF, Steadman DW. Estimation and evidence in forensic anthropology: sex and race. Am J Phys Anthropol. 2009;139(1):77-90.

10. Veyre-Goulet SA, Mercier C, Robin O, Guerin C. Recent human sexual dimorphism study using cephalometric plots on lateral teleradiography and discriminant function analysis. J Forensic Sci. 2008;53(4):786-9.

11. Spradley MK, Jantz RL. Sex estimation in forensic anthropology: skull versus poscranial elements. J Forensic Sci. 2011;56(2):289-96.

12. Galvão LCC. Identificação do sexo através de medidas cranianas [mestrado]. São Paulo: Faculdade de Odontologia de Piracicaba da Universidade Estadual de Campinas; 1994.

13. Saliba CA. Contribuição ao estudo do dimorfismo sexual através de medidas do crânio [mestrado]. São Paulo: Faculdade de Odontologia de Piracicaba da Universidade Estadual de Campinas;1999.

14. Sampaio CMA. Investigação do sexo por mensurações crânio-faciais. [mestrado] São Paulo: Faculdade de Odontologia de Piracicaba da Universidade Estadual de Campinas; 1999. 
15. Saini V, Srivastava R, Rai RK, Shamal SN, Singh TB, Tripathi SK. Mandibular ramus: na indicator for sex in fragmentary mandible. J Forensic Sci. 2011;56 Suppl 1:13-6.

16. Ikeda T, Nakamurs M, Itoh M. Sex differences in the zygomatic angle in japanese patients analyzed by MRI. Aesthetic Plast surg. 1999;23(5):349-53.

17. Steyn M, Iscan MY. Sexual dimorphism in crania and mandibles of South African Whites. Forensic Sci Int. 1998;98(1-2):9-16.

18. Almeida Jr. E, Galvão LCC, Flores PSC, Araújo TM. Investigação do sexo através de uma área triangular facial formada pela interseção dos pontos: forame infraorbital direito, esquerdo e o próstio, em crânios secos de adultos. Rev. ciênc. méd. biol. 2010;9(1):8-12. 